International Journal of Pure and Applied Mathematics

Volume 88 No. 1 2013, 77-90

ISSN: 1311-8080 (printed version); ISSN: 1314-3395 (on-line version)

url: http://www.ijpam.eu

doi: http://dx.doi.org/10.12732/ijpam.v88i1.6

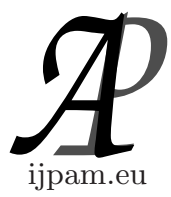

\title{
COMMON FIXED POINT THEOREMS FOR CONTRACTION MAPPINGS USING WEAK RECIPROCAL CONTINUITY
}

\author{
Saurabh Manro ${ }^{1}$, Sanjay Kumar ${ }^{2}$, \\ Satwinder Singh Bhatia ${ }^{3}$, Shin Min Kang ${ }^{4}$ \\ ${ }^{1,3}$ School of Mathematics and Computer Applications \\ Thapar University \\ Patiala, 147004, Punjab, INDIA \\ ${ }^{2}$ Department of Mathematics \\ Deenbandhu Chhotu Ram University of Science and Technology \\ Murthal, 131039, Sonepat, INDIA \\ ${ }^{4}$ Department of Mathematics and RINS \\ Gyeongsang National University \\ Jinju, 660-701, KOREA
}

\begin{abstract}
In this paper, we prove common fixed point theorems for contraction mappings using weak reciprocal continuity in metric spaces and Menger spaces.
\end{abstract}

AMS Subject Classification: 47H10, 54H 25

Key Words: weak reciprocal continuity, compatible, $R$-weakly commuting, $R$-weakly commuting of type $\left(A_{f}\right)$, of type $\left(A_{g}\right)$ and of type $(P)$

\section{Introduction and Preliminaries}

In 1922, Banach proved a common fixed point theorem, which ensures under appropriate conditions, the existence and uniqueness of a fixed point. This result of Banach is known as Banach's fixed point theorem or Banach Contraction

Received: June 26, 2013

(C) 2013 Academic Publications, Ltd.

$\S$ Correspondence author url: www.acadpubl.eu 
Principle. Many authors have extended, generalized and improved the Banach's fixed point theorem in different ways.

Jungck [3] proved a common fixed point theorem for commuting mappings, which generalizes the Banachs fixed point theorem. This Many aurthors has generalized this theorem, but suffers from one drawback that the continuity of mapping throughout the space.

Jungck [4] defined the concept of compatible mappings.

Definition 1.1. Let $f$ and $g$ be two self-mappings of a metric space $(X, d)$. Then $f$ and $g$ are is said to be compatible if $\lim _{n \rightarrow \infty} d\left(f g x_{n}, g f x_{n}=0\right.$ whenever $\left\{x_{n}\right\}$ is a sequence in $X$ such that $\lim _{n \rightarrow \infty} f x_{n}=g x_{n}=z$ for some $z \in X$.

In 1994, Pant [7] introduced the notion of (pointwise) $R$-weak commutativity in a metric space.

Definition 1.2. Let $f$ and $g$ be two self-mappings of a metric space $(X, d)$. Then $f$ and $g$ are said to be $R$-weakly commuting at a point $x \in X$ if there exists $R>0$ such that $d(f g x, g f x) \leq R d(f x, g x)$.

Definition 1.3. Let $f$ and $g$ be two self-mappings of a metric space $(X, d)$. Then $f$ and $g$ are called pointwise $R$-weakly commuting on $X$ if given $x \in X$, there exists $R>0$ such that $d(f g x, g f x) \leq R d(f x, g x)$.

In 1997, Pathak et al. [10] generalized the notion of $R$-weakly commuting mappings to $R$-weakly commuting mappings of type $\left(A_{f}\right)$ and of type $\left(A_{g}\right)$.

Definition 1.4. Let $f$ and $g$ be two self-mappings of a metric space $(X, d)$. Then $f$ and $g$ are called $R$-weakly commuting of type $\left(A_{g}\right)$ if there exists $R>0$ such that $d(f f x, g f x) \leq R d(f x, g x)$ for all $x \in X$.

Similarly, the two self-mappings $f$ and $g$ are called $R$-weakly commuting of type $\left(A_{f}\right)$ if there exists $R>0$ such that $d(f g x, g g x) \leq R d(f x, g x)$ for all $x \in X$.

It is obvious that pointwise $R$-weakly commuting mappings commute at their coincidence points and pointwise $R$-weak commutativity is equivalent to commutativity at coincidence points. It may be noted that both compatible and non-compatible mappings can be $R$-weakly commuting of type $\left(A_{g}\right)$ or $\left(A_{f}\right)$.

Definition 1.5. Let $f$ and $g$ be two self-mappings of a metric space $(X, d)$. Then $f$ and $g$ are called $R$-weakly commuting of type $(P)$ if there exists $R>0$ such that $d(f f x, g g x) \leq R d(f x, g x)$ for all $x \in X$.

In 1998, Pant [8] introduced a new continuity condition, known as reciprocal continuity and obtained a common fixed point theorem by using the compatibil- 
ity in a metric space and also showed that in the setting of common fixed point theorems for compatible mappings satisfying contraction conditions, the notion of reciprocal continuity is weaker than the continuity of one of the mappings. Also, the notion of pointwise $R$-weakly commuting mappings made the scope of the study of common fixed point theorems from the class of compatible to the wider class of pointwise $R$-weakly commuting mappings. Subsequently, several common fixed point theorems have been proved by combining the ideas of $R$ weakly commuting mappings and reciprocal continuity of mappings in different settings.

Definition 1.6. Let $f$ and $g$ be two self-mappings of a metric space $(X, d)$. Then $f$ and $g$ are called reciprocally continuous if $\lim _{n \rightarrow \infty} f g x_{n}=f z$ and $\lim _{n \rightarrow \infty} g f x_{n}=g z$, whenever $\left\{x_{n}\right\}$ is a sequence such that $\lim _{n \rightarrow \infty} f x_{n}=\lim _{n \rightarrow \infty} g x_{n}=$ $z$ for some $z \in X$.

If $f$ and $g$ are both continuous, then they are obvious reciprocally continuous but the converse is not true $([8])$.

Recently, Pant et al. [9] generalized the notion of reciprocal continuity to weak reciprocal continuity as follows:

Definition 1.7. Let $f$ and $g$ be two self-mappings of a metric space $(X, d)$. Then $f$ and $g$ are called weakly reciprocally continuous if $\lim _{n \rightarrow \infty} f g x_{n}=f z$ or $\lim _{n \rightarrow \infty} g f x_{n}=g z$, whenever $\left\{x_{n}\right\}$ is a sequence such that $\lim _{n \rightarrow \infty} f x_{n}=\lim _{n \rightarrow \infty} g x_{n}=$ $z$ for some $z \in X$.

If $f$ and $g$ are reciprocally continuous, then they are obviously weakly reciprocally continuous but the converse is not true ([9]). As an application of weak reciprocal continuity, we prove common fixed point theorems under contractive conditions that extend the scope of the study of common fixed point theorems from the class of compatible continuous mappings to a wider class of mappings which also includes non-compatible and discontinuous mappings.

An essential feature of metric spaces is that for any two points in a metric space, a positive number called the distance between two points is defined. One generalization of a metric space is initiated by Menger [5]. The concept of a probabilistic metric space corresponds to situations when we don't know the distance between the points, i.e., the distance between the points is inexact.

In 1942, Menger [5] introduced the notion of a probabilistic metric space as a generalization of a metric space which is defined as below.

A real valued function $f(x)$ defined on the set of real numbers is called a distribution function if it is non-decreasing, left-continuous with inf $f(x)=0$ and $\sup f(x)=1$. 
Let $H$ denotes the distribution function defined as follows.

$$
H(x)= \begin{cases}0 & \text { if } x \leq 0 \\ 1 & \text { if } x>0\end{cases}
$$

Definition 1.8. A probabilistic metric space (briefly, PM-space) is a pair $(X, F)$, where $X$ is a set and $F$ is a function defined on $X \times X$ into the set of distribution functions satisfying for all $x, y, z \in X$ and $s, t>0$.

(PM1) $F(x, y ; 0)=0$,

(PM2) $F(x, y ; t)=H(t)$ if and only if $x=y$,

(PM3) $F(x, y ; t)=F(y, x ; t)$,

$(\mathrm{PM} 4)$ if $F(x, y ; s)=1$ and $F(y, z ; t)=1$, then $F(x, z ; s+t)=1$.

For each $x, y \in X$ and for each real number $t>0, F(x, y ; t)$ is to be thought of as the probability that the distance between $x$ and $y$ isless then $t$. Of course, any metric space $(X, d)$ may be regarded as a PM-space. Indeed, if $(X, d)$ is a metric space, then the distribution function $F(x, y ; t)$ defined by $F(x, y ; t)=H(t-d(x, y))$ induces a PM-space.

Definition 1.9. ([11]) A $t$-norm is a binary operation $\Delta:[0,1] \times[0,1] \rightarrow$ $[0,1]$ satisfying for all $a, b, c, d \in[0,1]$

(t1) $\Delta(0,0)=0$

(t2) $\Delta(0,1)=1$

(t3) $\Delta(a, b)=\Delta(b, a)$,

(t4) if $a \leq c$ and $b \leq d$, then $\Delta(a, b) \leq \Delta(c, d)$,

(t5) $\Delta(\Delta(a, b), c)=\Delta(a, \Delta(b, c))$.

Examples of $t$-norm are $\Delta(a, b)=\min \{a, b\}$ and $\Delta(a, b)=a b$.

Definition 1.10. A Menger PM-space (briefly, Menger space) is a triple $(X, F, \Delta)$, where $(X, F)$ is a PM-space and $\Delta$ is a $t$-norm satisfying

(PM5) $F(x, z ; s+t) \geq \Delta(F(x, y ; s), F(y, z ; t))$ for all $x, y, z \in X$ and $s, t>0$.

This inequality is known as Menger's triangle inequality. The development of fixed point theory in PM-spaces was due to Schweizer and Sklar [11]. Sehgal and Bharucha-Reid [12] initiated the study of contraction mapping theorems in PM-spaces. Subsequently, several contraction mapping theorems for commuting mappings have been proved in PM-spaces.

Definition 1.11. ([2], [6]) Let $f$ and $g$ be two self-mappings of a Menger space $(X, F, \Delta)$. Then $f$ and $g$ are said to be compatible if $\lim _{n \rightarrow \infty} F\left(f g x_{n}, g f x_{n} ; t\right)$ $=1$ whenever $\left\{x_{n}\right\}$ is a sequence in $X$ such that $\lim _{n \rightarrow \infty} f x_{n}=\lim _{n \rightarrow \infty} g x_{n}=z$ for some $z \in X$. 
Definition 1.12. ([1]) Let $f$ and $g$ be two self-mappings of a Menger space $(X, F, \Delta)$. Then $f$ and $g$ are said to be $R$-weakly commuting at a point $x \in X$ if there exists $R>0$ such that $F(f g x, g f x ; t) \geq F(f x, g x ; t / R)$ for all $t>0$.

Definition 1.13. ([1]) Let $f$ and $g$ be two self-mappings of a Menger space $(X, F, \Delta)$. Then $f$ and $g$ are called pointwise $R$-weakly commuting on $X$ if given $x \in X$ there exists $R>0$ such that $F(f g x, g f x ; t) \geq F(f x, g x ; t / R)$ for all $t>0$.

Definition 1.14. Let $f$ and $g$ be two self-mappings of a Menger space $(X, F, \Delta)$. Then $f$ and $g$ are called $R$-weakly commuting of type $\left(A_{g}\right)$ if there exists $R>0$ such that $F(f f x, g f x ; t) \geq F(f x, g x ; t / R)$ for all $x \in X$ and $t>0$.

Similarly, the two self-mappings $f$ and $g$ are called $R$-weakly commuting of type $\left(A_{f}\right)$ if there exists some $R>0$ such that $F(f g x, g g x ; t) \geq F(f x, g x ; t / R)$ for all $x \in X$ and $t>0$.

Definition 1.15. Let $f$ and $g$ be two self-mappings of a Menger space $(X, F, \Delta)$. Then $f$ and $g$ are called $R$-weakly commuting of type $\left(A_{g}\right)$ if there exists $R>0$ such that $F(f f x, g g x ; t) \geq F(f x, g x ; t / R)$ for all $x \in X$ and $t>0$.

For our main results, we need the following lemma.

Lemma 1.16. ([11], [12]) Let $(X, F, \Delta)$ be a Menger space. If there exists a number $k \in(0,1)$ such that $F(x, y ; k t) \geq F(x, y ; t)$ for all $x, y \in X$ and $t>0$, then $x=y$.

Lemma 1.17. ([11]) Let $(X, F, \Delta)$ be a Menger space and $\left\{y_{n}\right\}$ be a sequence in $X$. If there exists a number $k \in(0,1)$ such that $F\left(y_{n}, y_{n+1} ; k t\right) \geq$ $F\left(y_{n-1}, y_{n} ; t\right)$ for all $n \in \mathbb{N}$ and $t>0$, then $\left\{y_{n}\right\}$ is a Cauchy sequence in $X$.

\section{Fixed Point Theorems in a Metric Space}

Now, we prove a fixed point theorem in a metric space.

Theorem 2.1. Let $f$ and $g$ be weakly reciprocally continuous self-mappings of a complete metric space $(X, d)$ satisfying

(C1) $f(X) \subset g(X)$,

$(C 2)$ there exists a number $h \in(0,1)$ such that

$$
d(f x, f y) \leq h \max \{d(g x, g y), d(f x, g x), d(f y, g y)\}
$$

for any $x, y \in X$. 
If $f$ and $g$ are either compatible or $R$-weakly commuting of type $\left(A_{g}\right)$ or $R$-weakly commuting of type $\left(A_{f}\right)$ or $R$-weakly commuting of type $(P)$, then $f$ and $g$ have a unique common fixed point.

Proof. Let $x_{0}$ be any point in $X$. Then as $f(X) \subset g(X)$, we can define a sequence $\left\{y_{n}\right\}$ in $X$ as

$$
y_{n}=f x_{n}=g x_{n+1} .
$$

Now, we show that $\left\{y_{n}\right\}$ is a Cauchy sequence in $X$. For proving this, by $(C 2)$, we have

$$
\begin{aligned}
d\left(y_{n}, y_{n+1}\right) & =d\left(f x_{n}, f x_{n+1}\right) \\
& \leq h \max \left\{d\left(g x_{n}, g_{n+1}\right), d\left(f x_{n}, g x_{n}\right), d\left(f x_{n+1}, g_{n+1}\right)\right\} \\
& =h \max \left\{d\left(y_{n-1}, y_{n}\right), d\left(y_{n}, y_{n-1}\right), d\left(y_{n+1}, y_{n}\right)\right\}
\end{aligned}
$$

If $d\left(y_{n}, y_{n+1}\right)>d\left(y_{n-1}, y_{n}\right)$, then we have

$$
d\left(y_{n}, y_{n+1}\right) \leq h d\left(y_{n}, y_{n+1}\right),
$$

which is a contraction. Thus we have

$$
d\left(y_{n}, y_{n+1}\right) \leq h d\left(y_{n-1}, y_{n}\right)
$$

Further we obtain

$$
d\left(y_{n}, y_{n+1}\right) \leq h d\left(y_{n-1}, y_{n}\right) \leq h^{2} d\left(y_{n-2}, y_{n-1}\right) \leq \cdots \leq h^{n} d\left(y_{0}, y_{1}\right)
$$

Therefore, for all $n, m \geq \mathbb{N}$ (: set of natural numbers), $n<m$, we have

$$
\begin{aligned}
d\left(y_{n}, y_{m}\right) \leq & d\left(y_{n}, y_{n+1}\right)+d\left(y_{n+1}, y_{n+2}\right)+d\left(y_{n+2}, y_{n+3}\right) \\
& +\cdots+d\left(y_{m-1}, y_{m}\right) \\
\leq & \left(h^{n}+h^{n+1}+h^{n+2}+\cdots+h^{m-1}\right) d\left(y_{0}, y_{1}\right) \\
\leq & \left(h^{n}+h^{n+1}+h^{n+2}+\cdots\right) d\left(y_{0}, y_{1}\right) \\
= & \frac{h^{n}}{1-h} d\left(y_{0}, y_{1}\right) \rightarrow 0
\end{aligned}
$$

as $n \rightarrow \infty$. Thus $\left\{y_{n}\right\}$ is a Cauchy sequence in $X$. As $X$ is complete, there exists a point $z$ in $X$ such that $\lim _{n \rightarrow \infty} y_{n}=z$. Therefore, by (2.1), we have

$$
\lim _{n \rightarrow \infty} y_{n}=\lim _{n \rightarrow \infty} f x_{n}=\lim _{n \rightarrow \infty} g x_{n+1}=z .
$$


Suppose that $f$ and $g$ are compatible mappings. Now, by weak reciprocal continuity of $f$ and $g$ implies that $\lim _{n \rightarrow \infty} f g x_{n}=f z$ or $\lim _{n \rightarrow \infty} g f x_{n}=g z$.

Let $\lim _{n \rightarrow \infty} g f x_{n}=g z$. Then compatibility of $f$ and $g$ gives

$$
\lim _{n \rightarrow \infty} d\left(f g x_{n}, g f x_{n}\right)=0,
$$

that is, $\lim _{n \rightarrow \infty} d\left(f g x_{n}, g z\right)=0$. Hence $\lim _{n \rightarrow \infty} f g x_{n}=g z$. By (2.1), we get

$$
\lim _{n \rightarrow \infty} f g x_{n+1}=\lim _{n \rightarrow \infty} f f x_{n}=g z .
$$

Therefore, by $(C 2)$, we get

$$
d\left(f z, f f x_{n}\right) \leq h \max \left\{d\left(g z, g f x_{n}\right), d(f z, g z), d\left(f f x_{n}, g f x_{n}\right)\right\} .
$$

As $n \rightarrow \infty$, since $h<1$

$$
d(f z, g z) \leq h d(f z, g z)
$$

so that, we get $f z=g z$. Again compatibility of $f$ and $g$ implies commutativity at a coincidence point. Hence $g f z=f g z=f f z=g g z$. Using $(C 2)$ we obtain

$$
\begin{aligned}
d(f z, f f z) & \leq h \max \{d(g z, g f z), d(f z, g z), d(f f z, g f z)\} \\
& =h d(f z, f f z),
\end{aligned}
$$

which implies that $f z=f f z$. Hence $f z=f f z=g f z$ and $f z$ is a common fixed point of $f$ and $g$.

Next suppose that $\lim _{n \rightarrow \infty} f g x_{n}=f z$. Then by $f(X) \subset g(X)$, there exists $u \in X$ such that $f z=g u$ and $\lim _{n \rightarrow \infty} f g x_{n}=g u$. Compatibility of $f$ and $g$ implies $\lim _{n \rightarrow \infty} g f x_{n}=g u$. By virtue of (2.1), this gives $\lim _{n \rightarrow \infty} f g x_{n+1}=\lim _{n \rightarrow \infty} f f x_{n}=g u$. Using $(C 2)$, we get

$$
d\left(f u, f f x_{n}\right) \leq h \max \left\{d\left(g u, g f x_{n}\right), d(f u, g u), d\left(f f x_{n}, g f x_{n}\right)\right\} .
$$

As $n \rightarrow \infty$,

$$
d(f u, g u) \leq h d(f u, g u)
$$

so that get $f u=g u$. Compatibility of $f$ and $g$ yields $f g u=g g u=f f u=g f u$. Finally, using $(C 2)$, we obtain

$$
\begin{aligned}
d(f u, f f u) & \leq h \max \{d(g u, g f u), d(f u, g u), d(f f u, g f u)\} \\
& =h d(f u, f f u)
\end{aligned}
$$


that is, $f u=f f u$. Hence $f u=f f u=g f u$ and $f u$ is a common fixed point of $f$ and $g$.

Now, suppose that $f$ and $g$ are $R$-weakly commuting of type $\left(A_{g}\right)$. Now, weak reciprocal continuity of $f$ and $g$ implies that $\lim _{n \rightarrow \infty} f g x_{n}=f z$ or $\lim _{n \rightarrow \infty} g f x_{n}=$ $g z$.

Assume that $\lim _{n \rightarrow \infty} g f x_{n}=g z$. Then $R$-weak commutativity of type $\left(A_{g}\right)$ of $f$ and $g$ yields $d\left(f f x_{n}, g f x_{n}\right) \leq R d\left(f x_{n}, g x_{n}\right)$, that is, $\lim _{n \rightarrow \infty} d\left(f f x_{n}, g z\right)=0$. This gives $\lim _{n \rightarrow \infty} f f x_{n}=g z$. Also, using $(C 2)$, we get

$$
d\left(f z, f f x_{n}\right) \leq h \max \left\{d\left(g z, g f x_{n}\right), d(f z, g z), d\left(f f x_{n}, g f x_{n}\right)\right\} .
$$

As $n \rightarrow \infty$,

$$
d(f z, g z) \leq h d(f z, g z)
$$

so that we get $f z=g z$. Again, by using $R$-weak commutativity of type $\left(A_{g}\right)$,

$$
d(f f z, g f z) \leq h d(f z, g z)=0 .
$$

This yields $f f z=g f z$. Therefore $f f z=f g z=g f z=g g z$. Using $(C 2)$, we get

$$
\begin{aligned}
d(f z, f f z) & \leq h \max \{d(g z, g f z), d(f z, g z), d(f f z, g f z\} \\
& =h d(f z, f f z),
\end{aligned}
$$

that is, we have $f z=f f z$. Hence $f z=f f z=g f z$ and $f z$ is a common fixed point of $f$ and $g$.

Similarly, if $\lim _{n \rightarrow \infty} f g x_{n}=f z$, then we can easily prove.

Suppose that $f$ and $g$ are $R$-weakly commuting of type $\left(A_{f}\right)$. Again, as done above, we can easily prove that $f z$ is a common fixed point of $f$ and $g$.

Finally, suppose $f$ and $g$ are $R$-weakly commuting of type $(P)$. Weak reciprocal continuity of $f$ and $g$ implies that $\lim _{n \rightarrow \infty} f g x_{n}=f z$ or $\lim _{n \rightarrow \infty} g f x_{n}=g z$.

Let us assume that $\lim _{n \rightarrow \infty} g f x_{n}=g z$. Then $R$-weak commutativity of type $(P)$ of $f$ and $g$ yields $d\left(f f x_{n}, g g x_{n}\right) \leq R d\left(f x_{n}, g x_{n}\right)$, that is, $\lim _{n \rightarrow \infty} d\left(f f x_{n}, g g x_{n}\right)=$ 0 . Using (2.1), we have $g f x_{n-1}=g g x_{n} \rightarrow g z$ and $f f x_{n} \rightarrow g z$ as $n \rightarrow \infty$. Also, using $(C 2)$ we get

$$
d\left(f z, f f x_{n}\right) \leq h \max \left\{d\left(g z, g f x_{n}\right), d(f z, g z), d\left(f f x_{n}, g f x_{n}\right)\right\} .
$$

As $n \rightarrow \infty$,

$$
d(f z, g z) \leq h d(f z, g z)
$$


so that $f z=g z$. Again, by using $R$-weak commutativity of type $(P), d(f f z, g g z)$ $\leq R d(f z, g z)=0$. This yields $f f z=g g z$. Therefore $f f z=f g z=g f z=g g z$. Using $(C 2)$, we get

$$
\begin{aligned}
d(f z, f f z) & \leq h \max \{d(g z, g f z), d(f z, g z), d(f f z, g f z)\} \\
& =h d(f z, f f z)
\end{aligned}
$$

so that $f z=f f z$. Hence $f z=f f z=g f z$ and $f z$ is a common fixed point of $f$ and $g$.

Similarly, if $\lim _{n \rightarrow \infty} f g x_{n}=f z$, then we can easily prove.

Uniqueness of the common fixed point theorem follows easily in each of the four cases by using $(C 2)$. This completes the proof.

Next, we give an example to illustrate Theorem 2.1.

Example 2.2. ([9]) Let $(X, d)$ be a metric space, where $X=[2,20]$ and $d(x, y)=|x-y|$ for all $x, y \in X$. Define $f, g: X \rightarrow X$ by

$$
\left\{\begin{array} { l l } 
{ f x = 2 \quad \text { if } x = 2 \text { or } x > 5 , } \\
{ f x = 6 } & { \text { if } 2 < x \leq 5 , }
\end{array} \quad \left\{\begin{array}{ll}
g x=2 & \text { if } x=2, \\
g x=12 & \text { if } 2<x \leq 5, \\
g x=(x+1) / 3 & \text { if } x>5
\end{array}\right.\right.
$$

Then it can be verified that $f$ and $g$ satisfy the contraction condition $(C 2)$ with $2 / 5 \leq h<1$.

Let $\left\{x_{n}\right\}$ be a sequence in $X$ such that either $x_{n}=2$ or $x_{n}=5+1 / n$ for each $n$. Then $f$ and $g$ satisfy all the conditions of Theorem 2.1 and have a unique common fixed point at $x=2$.

\section{Fixed Point Theorems in a Menger Space}

Now, we prove a fixed point theorem in a Menger space.

Theorem 3.1. Let $f$ and $g$ be weakly reciprocally continuous self-mappings of a complete Menger space $(X, F, \Delta)$ satisfying

$(C 1) f X) \subset g(X)$,

$(C 3)$ there exists a number $k \in(0,1)$ such that

$$
F(f x, f y ; k t) \geq \min \{F(g x, g y ; t), F(f x, g x ; t), F(f y, g y ; t)\}
$$

for any $x, y \in X$ and $t>0$. If $f$ and $g$ are either compatible or $R$-weakly commuting of type $\left(A_{g}\right)$ or $R$-weakly commuting of type $\left(A_{f}\right)$ or $R$-weakly commuting of type $(P)$, then $f$ and $g$ have a unique common fixed point. 
Proof. Let $x_{0}$ be any point in $X$. Then as $F(X) \subset g(X)$, we can define a sequence $\left\{y_{n}\right\}$ in $X$ as

$$
y_{n}=f x_{n}=g x_{n+1} .
$$

Now, we show that $\left\{y_{n}\right\}$ is a Cauchy sequence in $X$. For proving this, by (C3), we have

$$
\begin{aligned}
& F\left(y_{n}, y_{n+1} ; k t\right) \\
& =F\left(f x_{n}, f x_{n+1} ; k t\right) \\
& \geq \min \left\{F\left(g x_{n}, g x_{n+1} ; t\right), F\left(f x_{n}, g x_{n} ; t\right), F\left(f x_{n+1}, g x_{n+1} ; t\right)\right\} \\
& =\min \left\{F\left(y_{n-1}, y_{n} ; t\right), F\left(y_{n}, y_{n-1} ; t\right), F\left(y_{n+1}, y_{n} ; t\right)\right\} .
\end{aligned}
$$

If $F\left(y_{n}, y_{n+1} ; k t\right)<F\left(y_{n-1}, y_{n} ; t\right)$, then we have

$$
F\left(y_{n}, y_{n+1} ; k t\right)>F\left(y_{n}, y_{n+1} ; k t\right)
$$

which is a contraction. Thus we have

$$
F\left(y_{n}, y_{n+1} ; k t\right) \geq F\left(y_{n-1}, y_{n} ; t\right)
$$

Then, by Lemma 1.17, $\left\{y_{n}\right\}$ is a Cauchy sequence in $X$. As $X$ is complete, there exists a point $z$ in $X$ such that $\lim _{n \rightarrow \infty} y_{n}=z$. Therefore, by (3.1), we have

$$
\lim _{n \rightarrow \infty} y_{n}=\lim _{n \rightarrow \infty} f x_{n}=\lim _{n \rightarrow \infty} g x_{n+1}=z .
$$

Suppose that $f$ and $g$ are compatible mappings. Now, by weak reciprocal continuity of $f$ and $g$ implies that $\lim _{n \rightarrow \infty} f g x_{n}=f z$ or $\lim _{n \rightarrow \infty} g f x_{n}=g z$.

Let $\lim _{n \rightarrow \infty} g f x_{n}=g z$. Then compatibility of $f$ and $g$ gives

$$
\lim _{n \rightarrow \infty} F\left(f g x_{n}, g f x_{n} ; t\right)=1
$$

that is, $\lim _{n \rightarrow \infty} F\left(f g x_{n}, g z ; t\right)=1$. Hence $\lim _{n \rightarrow \infty} f g x_{n}=g z$. By (3.1), we get $\lim _{n \rightarrow \infty} f g x_{n+1}=\lim _{n \rightarrow \infty} f f x_{n}=g z$. Therefore, by $(C 3)$, we get

$$
F\left(f z, f f x_{n} ; k t\right) \geq \min \left\{F\left(g z, g f x_{n} ; t\right), F(f z, g z ; t), F\left(f f x_{n}, g f x_{n} ; t\right)\right\} .
$$

As $n \rightarrow \infty$,

$$
\begin{aligned}
F(f z, g z ; k t) & \geq \min \{F(g z, g z ; t), F(f z, g z ; t), F(g z, g z ; t)\} \\
& =F(f z, g z ; t) .
\end{aligned}
$$


Hence, by Lemma 1.16, we get $f z=g z$. Again compatibility of $f$ and $g$ implies commutativity at a coincidence point. Hence $g f z=f g z=f f z=g g z$. Using (C3) we obtain

$$
\begin{aligned}
F(f z, f f z ; k t) & \geq \min \{F(g z, g f z ; t), F(f z, g z ; t), F(f f z, g f z ; t)\} \\
& =F(f z, f f z ; t),
\end{aligned}
$$

that is, we get $f z=f f z$. Hence $f z=f f z=g f z$ and $f z$ is a common fixed point of $f$ and $g$.

Next suppose that $\lim _{n \rightarrow \infty} f g x_{n}=f z$. Then by $f(X) \subset g(X)$ there exists $u \in X$ such that $f z=g u$ and $\lim _{n \rightarrow \infty} f g x_{n}=g u$. Compatibility of $f$ and $g$ implies $\lim _{n \rightarrow \infty} g f x_{n}=g u$. By virtue of (3.1), this gives $\lim _{n \rightarrow \infty} f g x_{n+1}=\lim _{n \rightarrow \infty} f f x_{n}=g u$. Using $(C 3)$, we get

$$
F\left(f u, f f x_{n} ; k t\right) \geq \min \left\{F\left(g u, g f x_{n} ; t\right), F(f u, g u ; t), F\left(f f x_{n}, g f x_{n} ; t\right)\right\} .
$$

As $n \rightarrow \infty$,

$$
\begin{aligned}
F(f u, g u ; k t) & \geq \min \{F(g u, g u ; t), F(f u, g u ; t), F(g u, g u ; t)\} \\
& =F(f u, g u ; t)
\end{aligned}
$$

so that we get $f u=g u$. Compatibility of $f$ and $g$ yields $f g u=g g u=f f u=$ $g f u$. Finally, using $(C 3)$, we obtain

$$
\begin{aligned}
F(f u, f f u ; k t) & \geq \min \{F(g u, g f u ; t), F(f u, g u ; t), F(f f u, g f u ; t)\} \\
& =F(f u, f f u ; t),
\end{aligned}
$$

that is, $f u=f f u$. Hence $f u=f f u=g f u$ and $f u$ is a common fixed point of $f$ and $g$.

Now, suppose that $f$ and $g$ are $R$-weakly commuting of type $\left(A_{g}\right)$. Now, weak reciprocal continuity of $f$ and $g$ implies that $\lim _{n \rightarrow \infty} f g x_{n}=f z$ or $\lim _{n \rightarrow \infty} g f x_{n}=$ $g z$.

Assume that $\lim _{n \rightarrow \infty} g f x_{n}=g z$. Then $R$-weak commutativity of type $\left(A_{g}\right)$ of $f$ and $g$ yields $F\left(f f x_{n}, g f x_{n} ; t\right) \geq F\left(f x_{n}, g x_{n} ; t / R\right)$, that is,

$$
\lim _{n \rightarrow \infty} F\left(f f x_{n}, g z ; t\right) \geq F(z, z ; t / R)=1 .
$$

This gives $\lim _{n \rightarrow \infty} f f x_{n}=g z$. Also, using $(C 3)$ we get

$$
F\left(f z, f f x_{n} ; k t\right) \geq \min \left\{F\left(g z, g f x_{n} ; t\right), F(f z, g z ; t), F\left(f f x_{n}, g f x_{n} ; t\right)\right\} .
$$


As $n \rightarrow \infty$,

$$
\begin{aligned}
F(f z, g z ; k t) & \geq \min \left\{F(g z, g z ; t), F(f z, g z ; t), F\left(g z, g g f x_{n} ; t\right)\right\} \\
& =F(f z, g z ; t)
\end{aligned}
$$

so that by Lemma 1.16, we get $f z=g z$. Again, by using $R$-weak commutativity of type $\left(A_{g}\right), F(f f z, g f z ; t) \geq F(f z, g z ; t / R)=1$. This yields $f f z=g f z$. Therefore, $f f z=f g z=g f z=g g z$. Using $(C 3)$, we get

$$
\begin{aligned}
F(f z, f f z ; k t) & \geq \min \{F(g z, g f z ; t), F(f z, g z ; t), F(f f z, g f z ; t)\} \\
& =F(f z, f f z ; t),
\end{aligned}
$$

that is, we have $f z=f f z$. Hence $f z=f f z=g f z$ and $f z$ is a common fixed point of $f$ and $g$.

Similarly, if $\lim _{n \rightarrow \infty} f g x_{n}=f z$, then we can easily prove.

Suppose that $f$ and $g$ are $R$-weakly commuting of type $\left(A_{f}\right)$. Again, as done above, we can easily prove that $f z$ is a common fixed point of $f$ and $g$.

Finally, suppose that $f$ and $g$ are $R$-weakly commuting of type $(P)$. Weak reciprocal continuity of $f$ and $g$ implies that $\lim _{n \rightarrow \infty} f g x_{n}=f z$ or $\lim _{n \rightarrow \infty} g f x_{n}=g z$.

Assume that $\lim _{n \rightarrow \infty} g f x_{n}=g z$. Then $R$-weak commutativity of type $(P)$ of $f$ and $g$ yields $F\left(f f x_{n}, g g x_{n} ; t\right) \geq F\left(f x_{n}, g x_{n} ; t / R\right)$, that is,

$$
\lim _{n \rightarrow \infty} F\left(f f x_{n}, g g x_{n} ; t\right) \geq F(z, z ; t / R)=1 .
$$

This gives $\lim _{n \rightarrow \infty} F\left(f f x_{n}, g g x_{n} ; t\right)=1$. Using (3.1) we have $g f x_{n-1}=g g x_{n} \rightarrow g z$ and $f f x_{n} \rightarrow g z$ as $n \rightarrow \infty$. Also, using (C3) we get

$$
F\left(f z, f f x_{n} ; k t\right) \geq \min \left\{F\left(g z, g f x_{n} ; t\right), F(f z, g z ; t), F\left(f f x_{n}, g f x_{n} ; t\right)\right\} .
$$

As $n \rightarrow \infty$,

$$
\begin{aligned}
F(f z, g z ; k t) & \geq \min \{F(g z, g z ; t), F(f z, g z ; t), F(g z, g z ; t)\} \\
& =F(f z, g z ; t),
\end{aligned}
$$

that is, by Lemma 1.16, we get $f z=g z$. Again, by using $R$-weak commutativity of type $(P), F(f f z, g g z ; t) \geq F(f z, g z ; t / R)=1$. This yields $f f z=g g z$. Therefore, $f f z=f g z=g f z=g g z$. Using $(C 3)$, we get

$$
\begin{aligned}
F(f z, f f z ; k t) & \geq \min \{F(g z, g f z ; t), F(f z, g z ; t), F(f f z, g f z ; t)\} \\
& =F(f z, f f z ; t)
\end{aligned}
$$


so that we get $f z=f f z$. Hence $f z=f f z=g f z$ and $f z$ is a common fixed point of $f$ and $g$.

Similarly, if $\lim _{n \rightarrow \infty} f g x_{n}=f z$, then we can easily prove.

Uniqueness of the common fixed point theorem follows easily in each of the four cases by using $(C 3)$. This completes the proof.

Next, we give an example to illustrate Theorem 3.1.

Example 3.2. ([9]) Let $(X, F, t)$ be a Menger space, where $X=[2,20]$ and $F(x, y, t)=t /(t+|x-y|)$ for all $x, y \in X$. Let $f, g: X \rightarrow X$ as in the Example 2.2. Then it can be verified that $f$ and $g$ satisfy the contraction condition $(C 3)$ with $2 / 5 \leq k<1$.

Let $\left\{x_{n}\right\}$ be a sequence in $X$ such that either $x_{n}=2$ or $x_{n}=5+1 / n$ for each $n$. Then $f$ and $g$ satisfy all the conditions of Theorem 3.1 and have a unique common fixed point at $x=2$.

\section{References}

[1] Lj.B. Ćirić, M.M. Milovanović-Arandjelović, Common fixed point theorem for $R$-weak commuting mappings in Menger Spaces, J. Indian Acad. Math., 22, No. 2 (2000), 199-210.

[2] Y.J. Cho, P.P. Murthy, M. Stojakovic, Compatible mappings of type $(A)$ and common fixed points in Menger spaces, Commun. Korean Math. Soc., 7, No. 2 (1992), 325-339.

[3] G. Jungck, Commuting mappings and fixed points, Amer. Math. Monthly, 83, No. 4 (1976), 261-263, doi: 10.2307/2318216.

[4] G. Jungck, Compatible mappings and common fixed points, Int. J. Math. Math. Sci., 9, No. 4 (1986), 771-779, doi: 10.1155/S0161171286000935.

[5] K. Menger, Statistical metrics, Proc. Natl. Acad. Sci. USA, 28, No. 12 (1942), 535-537, doi: 10.1073/pnas.28.12.535.

[6] S.N. Mishra, Common fixed points of compatible mappings in PM-spaces, Math. Japon., 36, No. 2 (1991), 283-289.

[7] R.P. Pant, Common fixed points of non-commuting mappings, J. Math. Anal. Appl., 188, No. 2 (1994), 436-440, doi: 10.1006/jmaa.1994.1437. 
[8] R.P. Pant, Common fixed points of four mappings, Bull. Cal. Math. Soc., 90, No. 4 (1998) 281-286.

[9] R.P. Pant, R.K. Bisht, D. Arora, Weak reciprocal continuity and fixed point theorems, Ann. Univ. Ferrara, 57, No. 1 (2011), 181-190, doi: 10.1007/s11565-011-0119-3.

[10] H.K. Pathak, Y.J. Cho, S.M. Kang, Remarks of $R$-weakly commuting mappings and common fixed point theorems, Bull. Korean Math. Soc., 34, No. 2 (1997), 247-257.

[11] B. Schweizer, A. Sklar, Probabilistic Metric Spaces, North-Holland Series in Probability and Applied Mathematics, North-Holland Publishing Co., New York, 1983.

[12] V.M. Sehgal, A.T. Bharucha-Reid, Fixed points of contraction mappings on probabilistic metric spaces, Math. Systems Theory, 6 (1972), 97-102. 